\title{
Polymer Spherulitic Growth Kinetics Mediated by Nanoparticle Assemblies
}

Abdullah S. Altorbaq, ${ }^{\dagger}$ Andrew M. Jimenez, ${ }^{\dagger}$ Julia Pribyl, $\downarrow$ Brian Benicewicz,,+ Alejandro J. Müller, ${ }^{\circledR}+*$ Sanat K. Kumar ${ }^{\dagger} *$

$\dagger$ Department of Chemical Engineering, Columbia University, New York, New York, 10027, USA.

${ }^{\natural}$ Department of Chemistry and Biochemistry, University of South Carolina. Columbia, South Carolina 29208, USA.

${ }^{\S}$ POLYMAT and Faculty of Chemistry, Basque Country University UPV/EHU, Paseo Lardizabal 3, 20018, Donostia-San Sebastián, Spain.

${ }^{\ddagger}$ Ikerbasque, Basque Science Foundation, 48009, Bilbao, Spain.

Keywords. Polymer Nanocomposite, Dispersion, Crystallization, Spherulitic growth kinetics, Bimodal Brushes.

For table of content use only:

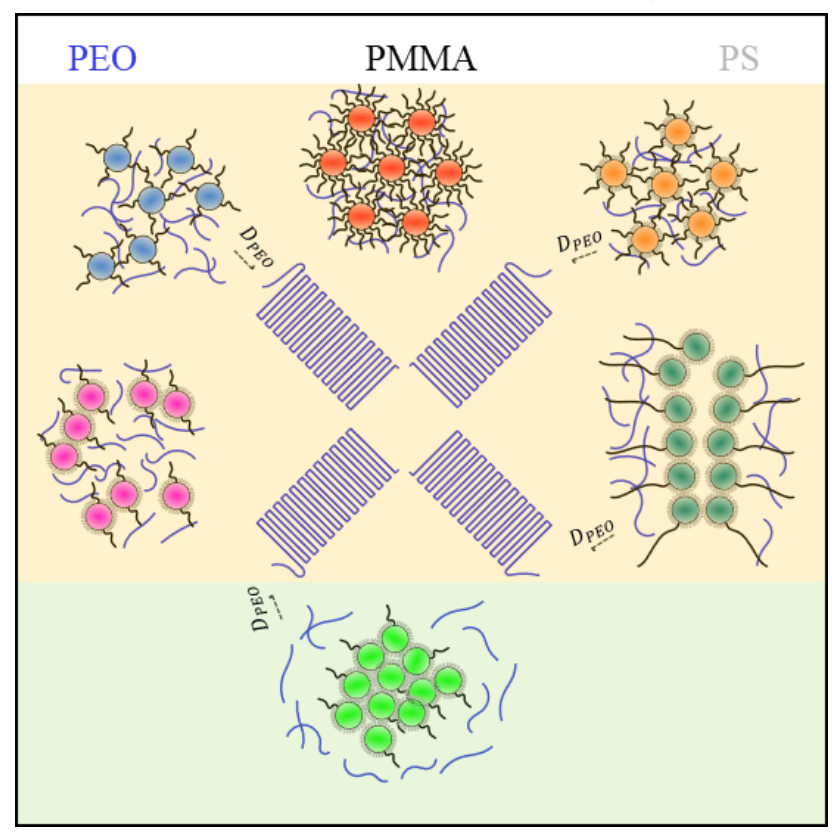




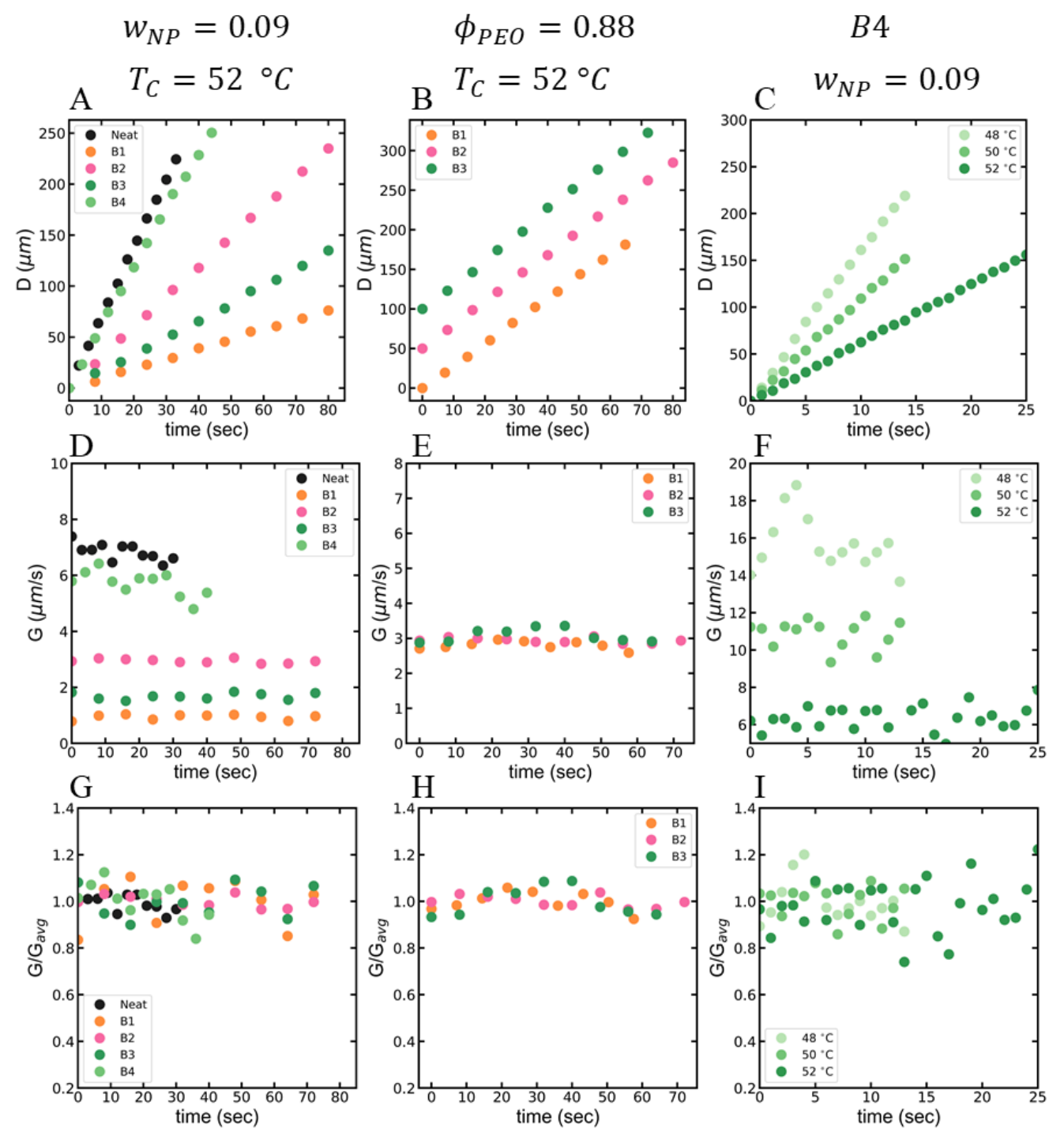

Figure S1: PLOM measurements for the neat PEO and bimodal composites showing spherulites' diameters $D$ (A $\mathrm{C})$, and their corresponding growth rates $G(\mathrm{D}-\mathrm{F})$ and normalized growth rates $G / G_{\text {Average }}(\mathrm{G}-\mathrm{I})$ as a function of time. Loadings, crystallization temperatures and sample names are either shown in the legend or the figure overhead.

Figure $\mathrm{S} 1$ is intended to show the linear growth regime over a long time just prior to spherulitic impingement for different NP self-assembled structures. This is postulated by the unchanged overall trend of $G / G_{\text {avg }}$ when plotted as a function of time. 

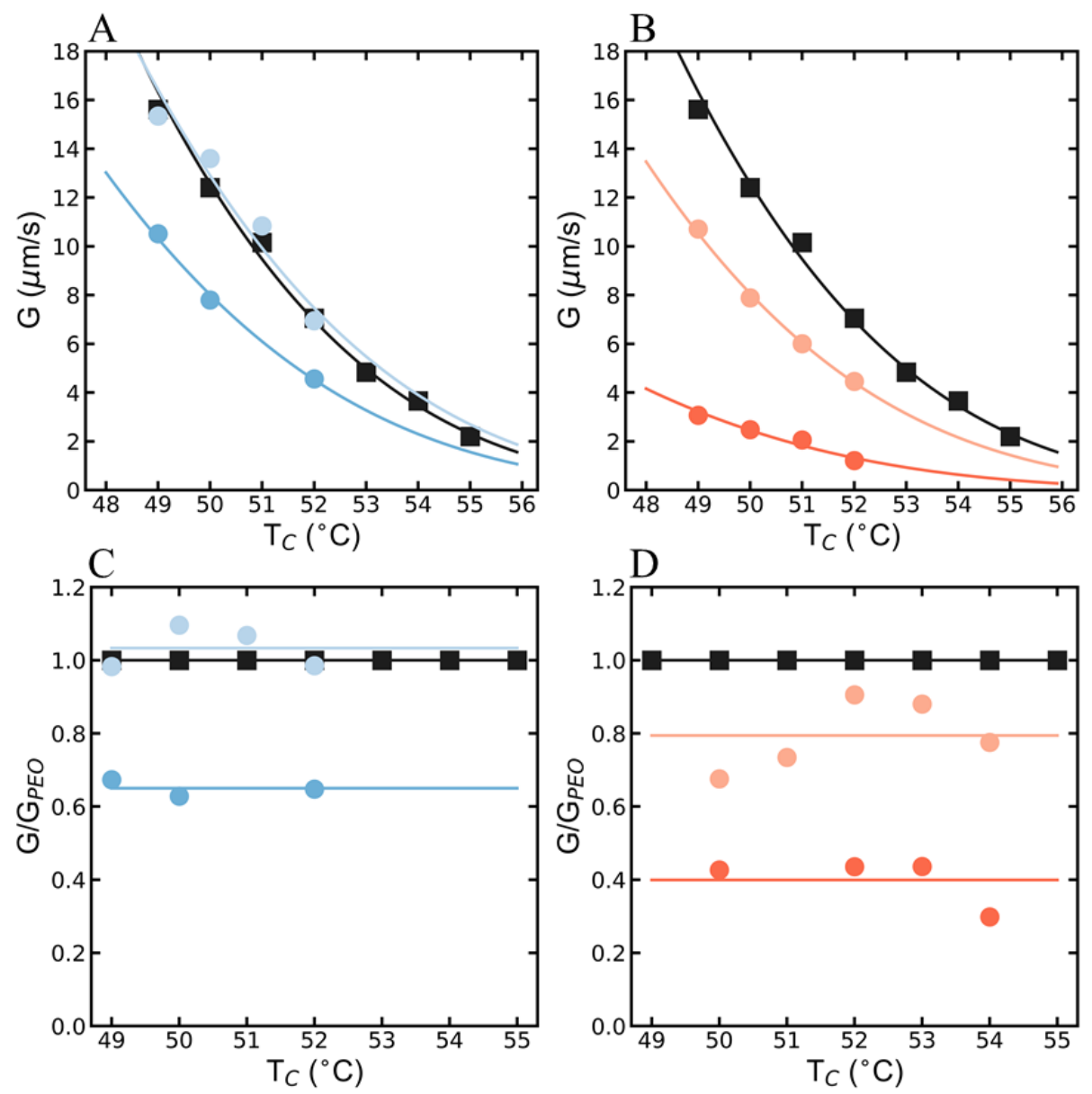

Figure S2: (A, B) spherulite growth rate of A1 and A2 unimodal systems, respectively. The solid lines correspond to Lauritzen-Hoffman fits. (C, D) composite growth rates normalized by neat PEO at each crystallization temperature. The solid lines represent the average values of the normalized growth rates. Low and medium NP loadings are plotted for each figure that corresponds to the color shading. 

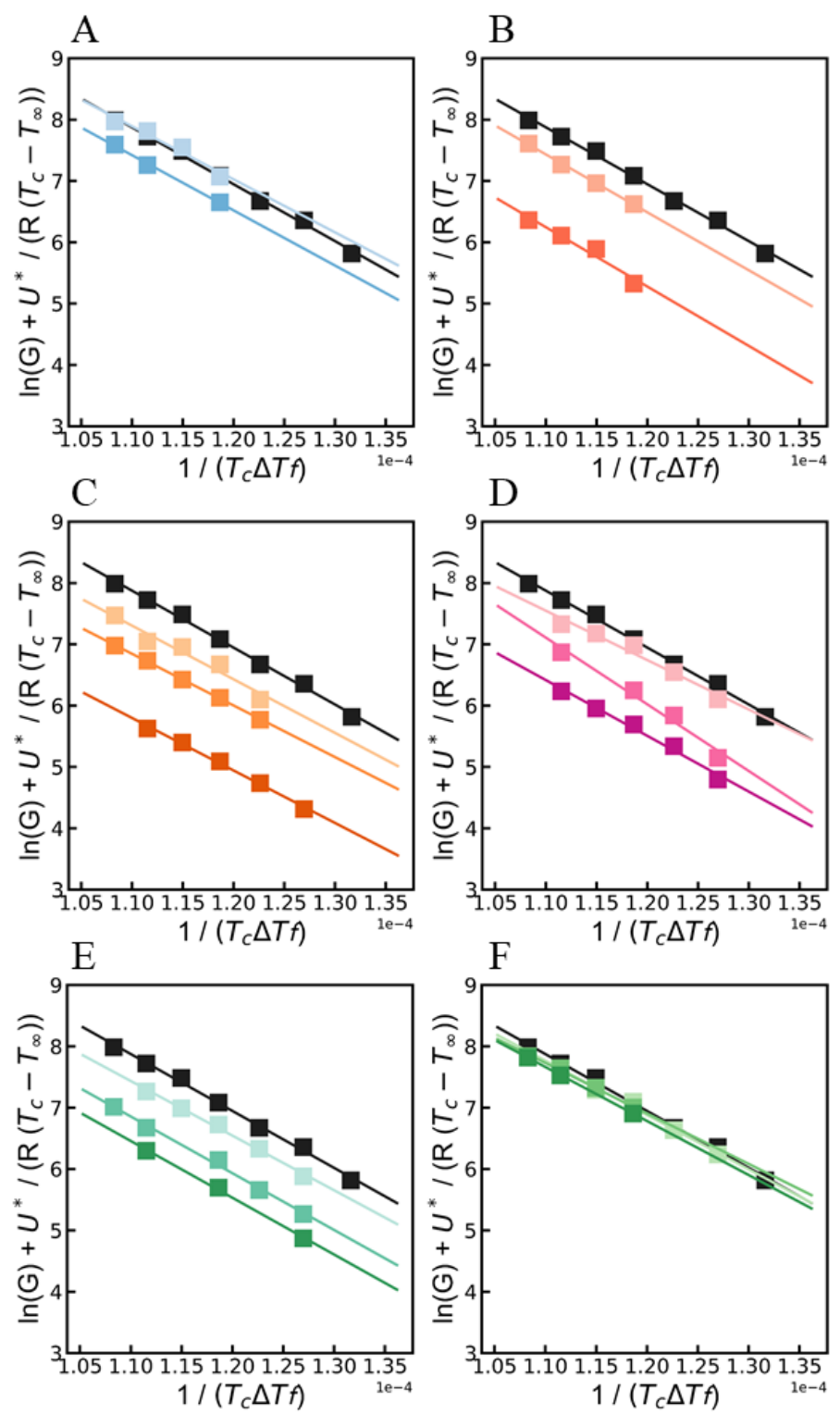

Figure S3: $(\mathrm{A}-\mathrm{F})$ the logarithmic values of the growth rates plotted in the form of LH fit showing the measured (squares) and fit (solid lines) values of the growth rate for A1, A2, B1, B2, B3, B4 systems, respectively. Neat PEO (black) is plotted in each subfigure for comparison. 
In addition to the LH fit, the following crystallization kinetics models were used to fit the experimental data: ${ }^{1}$

$G(T)=G_{0} \exp \left(\frac{E_{D}^{*}}{R T}-\frac{g_{2} T_{m}^{0}}{T \Delta T}\right)$

$G(T)=G_{0} \exp \left(\frac{E_{D}^{*}}{R T}-\frac{g_{3}\left(T_{m}^{0}\right)^{2}}{T(\Delta T)^{2}}\right)$

$E_{D}^{*}$ is the transport Arrhenius activation free energy for the molecules to diffuse into the nuclei. $g_{2}$ and $g_{3}$ are the two- and three-dimensional enthalpies of fusion per repeating unit, respectively. Figure S4 shows consistent independence of the secondary nucleation term as predicted from the LH fit. Thus, the effect on the growth is embodied into the transport of the chains. 

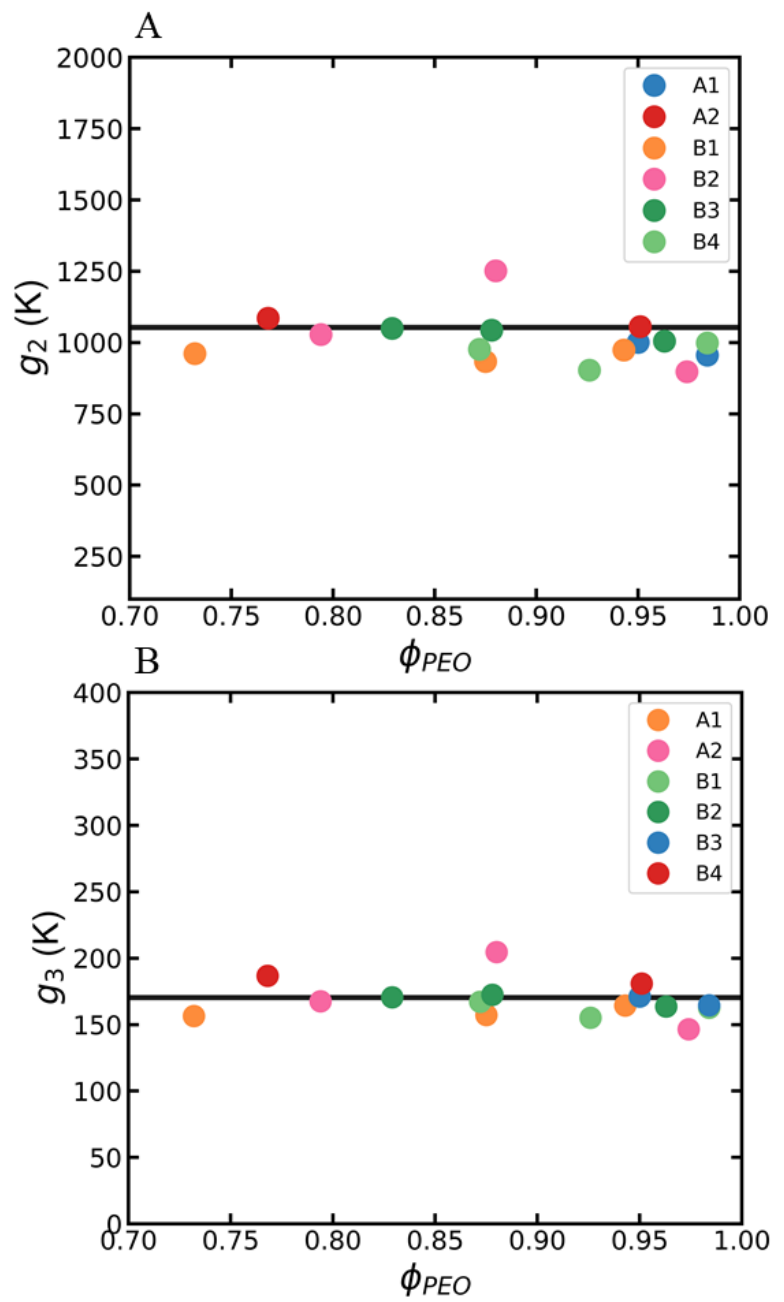

Figure S4: the fit values of $g_{2}(\mathrm{~A})$ and $g_{3}(\mathrm{~B})$ as a function of PEO volume fraction for unimodal and bimodal systems. Solid line corresponds to the values of the neat PEO. 

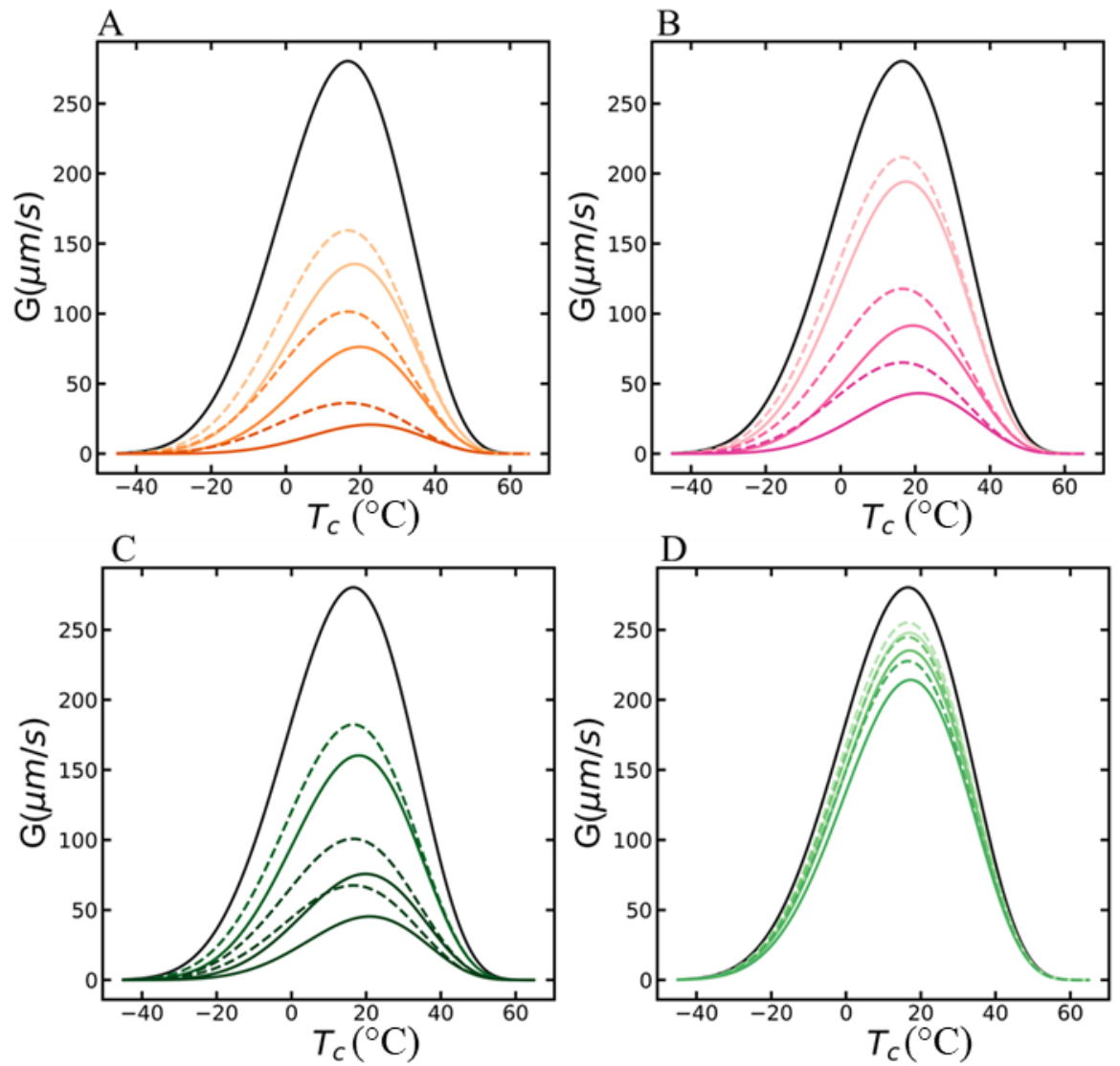

Figure S5: Hypothetical evaluated bell shape of the spherulitic growth by assuming a single spherulite growing in an infinite melt as a function of crystallization temperature $\mathrm{T}_{\mathrm{c}}\left({ }^{\circ} \mathrm{C}\right) . K_{g}$ values are considered to be equal to the neat PEO, where the change in the measured $G$ is attributed to the prefactor $G_{0}$ (dashed line) or the activation barrier $U^{*}$ (solid line). (A-D) correspond respectively to B1, B2, B3 and B4

It is notable from Figures $\mathrm{S} 5$ that attributing the growth reduction to either $G_{0}$ or $U^{*}$ is highly indistinguishable at our crystallization temperature range $\left(>48^{\circ} \mathrm{C}\right)$. 


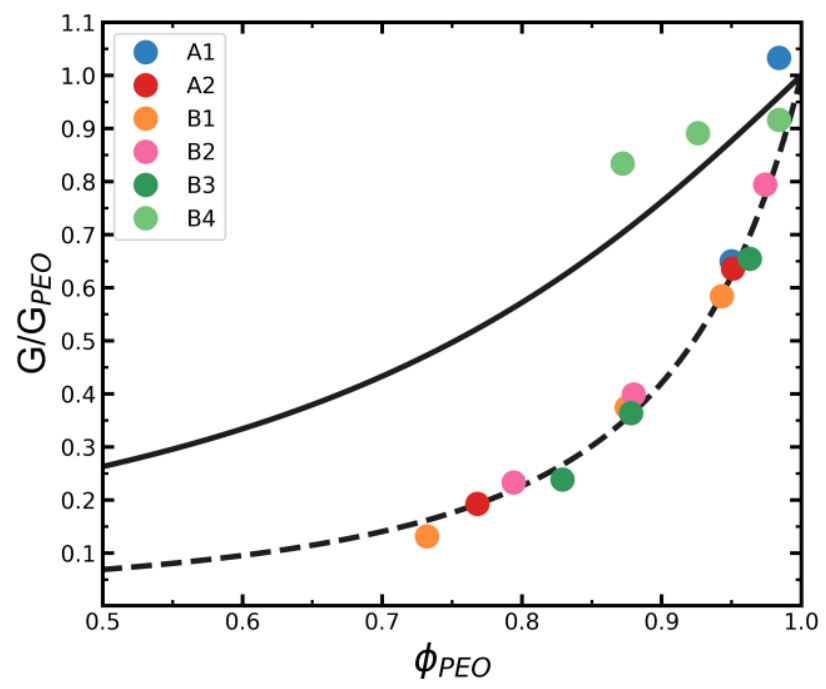

Figure S6: Average values of the normalized growth rates $G / G_{P E O}$ plotted as a function of PEO volume fraction. Solid and dash-dotted lines correspond to the Einstein-Batchelor and the parabolic $\left(1+10.5 \phi_{N P}+32.9{\phi_{N P}}^{2}\right)^{-1}$ fits, respectively. 

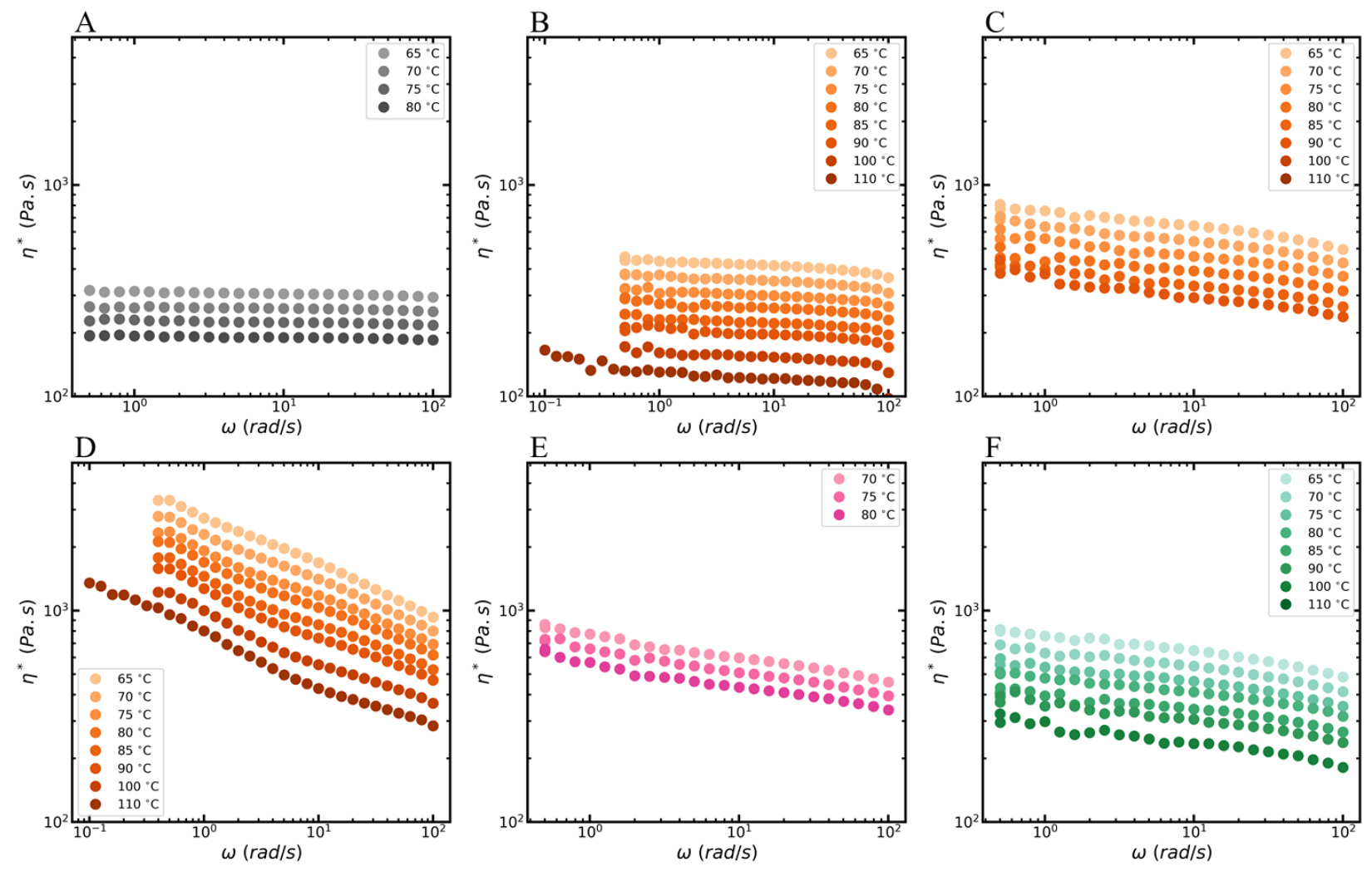

Figure S7: Complex viscosity as a function of frequency for different temperatures. (A) neat PEO, (B) low loading $\mathrm{B} 1\left[\phi_{P E O}=0.94\right],(\mathrm{C})$ medium loading B1 $\left[\phi_{P E O}=0.88\right]$, (D) high loading $\mathrm{B} 1\left[\phi_{P E O}=0.73\right],(\mathrm{E})$ medium loading $\mathrm{B} 2\left[\phi_{P E O}=0.88\right],(\mathrm{F})$ medium loading $\mathrm{B} 1\left[\phi_{P E O}=0.88\right]$. 

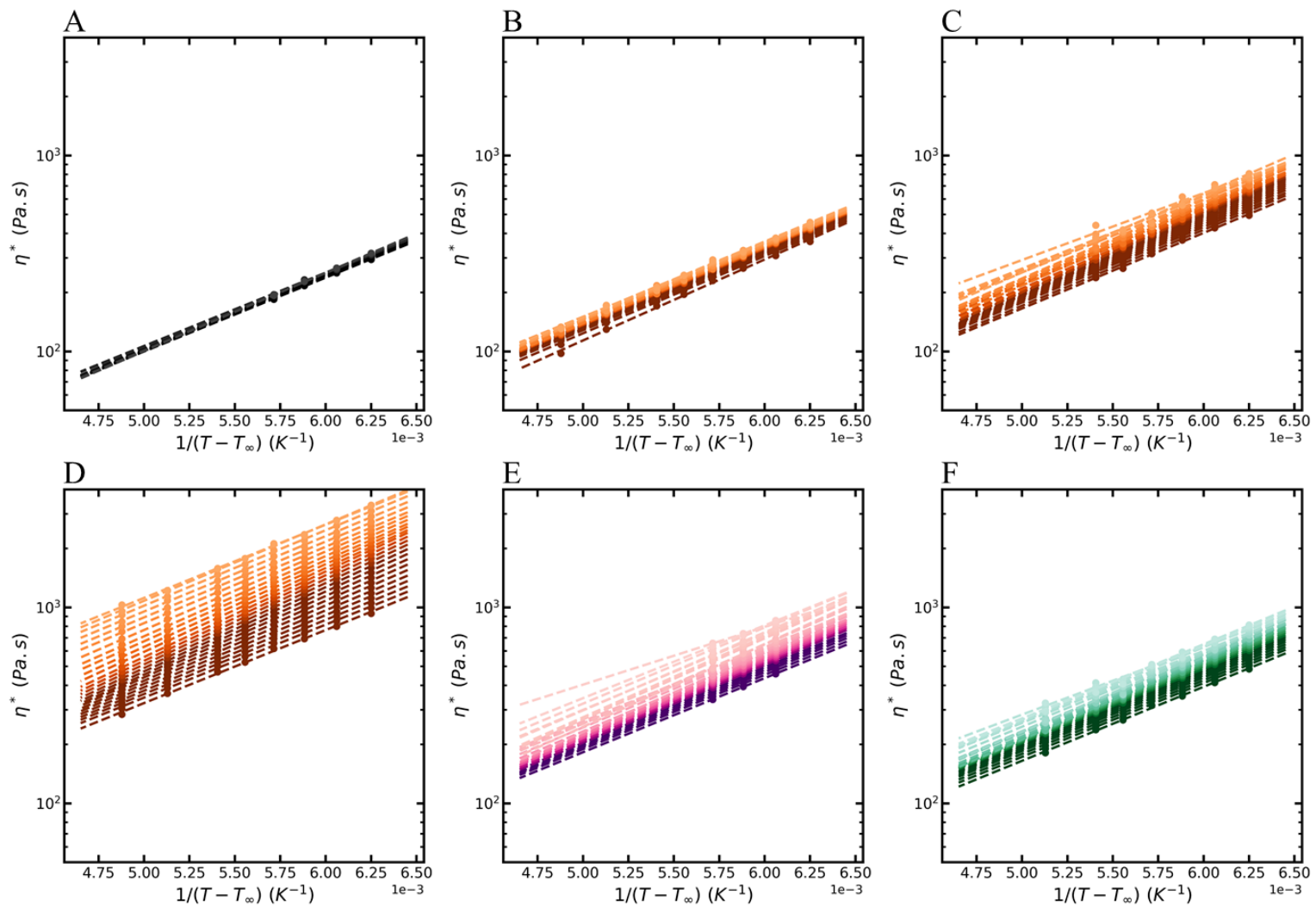

Figure S8: The complex viscosity, from Figure S7, replotted as a function of $\frac{1}{T-T_{\infty}}$ at different frequencies, where the dashed-lines correspond to the VFTH fit. Darker shadings correspond to higher frequencies.

It is important to note that the shift in the slope $\left(U^{*}\right)$ at a certain frequency, which is observed in figure 9B, is due to the change in the viscosity regime (frequency exponent). However, at higher frequency the exponent is constant resulting in constant values of $U^{*}$ for different loadings. 


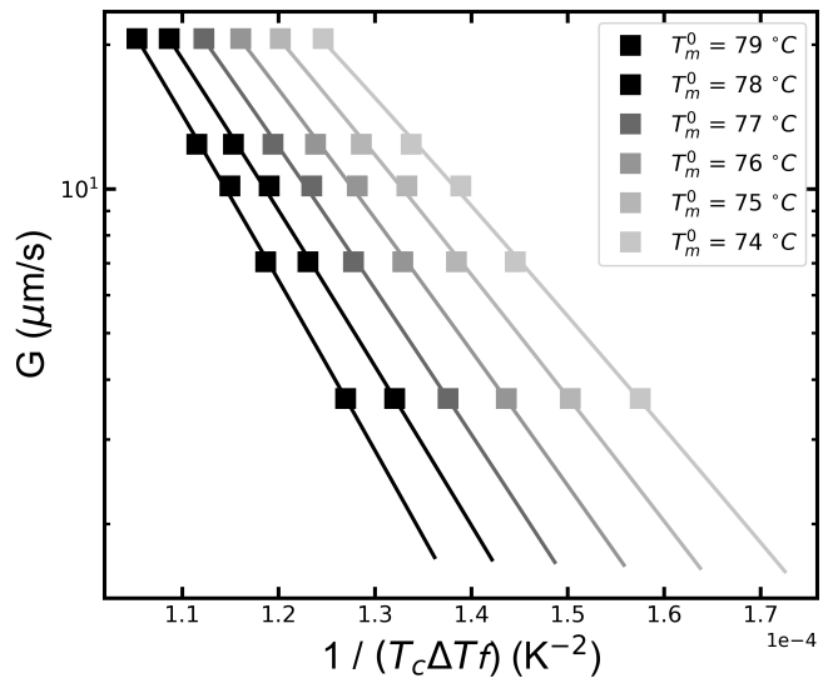

Figure S9: Growth rate of neat PEO as a function of $\frac{1}{T_{c} \Delta T f}$ using different values of $T_{m}^{0}$. Solid lines correspond to LH fit showing the change in the slope for these different equilibrium melting temperatures.

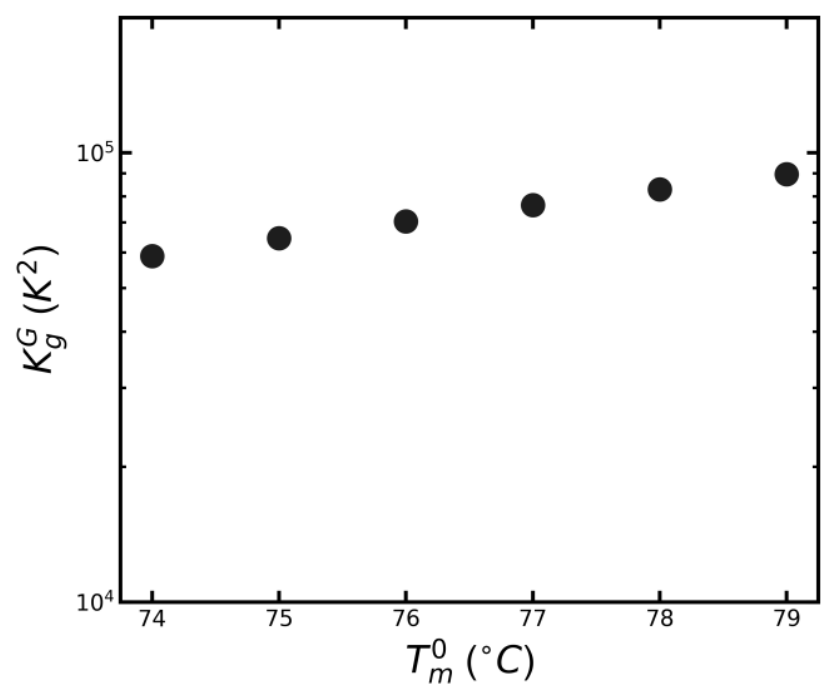


Figure S10: replotting $K_{g}^{G}$ from the LH fit in Figure S9 as a function of $T_{m}^{0}$.

The extrapolated equilibrium melting point for the PEO was measured to be $79{ }^{\circ} \mathrm{C} .{ }^{2}$ Based on previous non-isothermal crystallization experiments we found that the melting point was depressed by $\approx 5{ }^{\circ} \mathrm{C}$ for the largest NP loading examined. This is at the limit of uncertainties in our experiments, especially when coupled to long extrapolations required to obtain the equilibrium melting point from typically analyses (e.g., Hoffman-Weeks). On the other hand, the GibbsThomson based extrapolation yielded essentially a fixed equilibrium melting point. Because of these facts we reasoned that holding the melting point constant at the value of the pure PEO was the rational thing to do. If we included a maximum $5{ }^{\circ} \mathrm{C}$ depression of melting point for the largest loading then that lowers $\Delta \mathrm{T}$ by $15-25 \%$. This is the limit of sensitivity in our statements about the activation energy. 


\section{Reference}

(1) Lorenzo, A. T.; Müller, A. J. Estimation of the Nucleation and Crystal Growth Contributions to the Overall Crystallization Energy Barrier. J. Polym. Sci. Part B Polym. Phys. 2008, 46 (14), 1478-1487.

(2) Jimenez, A. M.; Altorbaq, A. S.; Müller, A. J.; Kumar, S. K. Polymer Crystallization Under Confinement by Well-Dispersed Nanoparticles. Macromolecules. 2020, 53, 1025610266. 